Pennsylvania, ${ }^{10}$ Kaiser Permanente Vaccine Study Center, Oakland, California, ${ }^{11}$ DaVita Medical Group, Colorado Springs, Colorado, ${ }^{12}$ CureVac AG, Tübingen, Germany

Session: 254. Vaccines for the Elderly and Immune Compromised Saturday, October 6, 2018: 12:30 PM

Background. Herpes zoster (HZ) and its related complications are associated with a significant burden of illness in older adults, which negatively impacts patients' physical functioning and quality-of-life $(\mathrm{QoL})$. The recombinant zoster vaccine (RZV) shows high efficacy for the prevention of $\mathrm{HZ}$ in older adults but is associated with local and systemic reactions. Therefore, this study assessed the impact of RZV reactogenicity upon the physical functioning and QoL of participants.

Methods. 401 adults aged $\geq 50$ years received a dose of RZV at 0 and 2 month in this open-label, single-arm, multicenter study (NCT02979639). Changes in mean SF-36 Physical Functioning score were assessed between pre-dose-1 vaccination and post-dose-1 vaccination for 7 days (primary endpoint). Decreased scores are associated with decreased physical functioning. QoL, reactogenicity and safety were also assessed. The current analysis was performed post-dose-1 vaccination of the 2-dose RZV schedule.

Results. No clinically meaningful reductions in overall mean SF-36 Physical Functioning scores from pre- to post-RZV dose- 1 were observed (mean +1.9 points) and no overall quality-adjusted-life-year loss was recorded post-dose- 1 . However, grade 3 reactogenicity occurred in $9.5 \%$ of participants, and was associated with a transient, clinically-important decrease in SF-36 Physical Functioning score (impacting activities such as walking, carrying groceries, climbing stairs) on Days 1-2 post-first-vaccination (Table 1). The solicited local symptoms were pain $(77.5 \%)$, redness $(23.0 \%)$ and swelling (13.3\%); the most frequent solicited systemic reactions were fatigue (33.5\%), headache $(28.3 \%)$ and myalgia $(26.8 \%)$.

Conclusion. Overall, the physical functioning and QoL of older adults were not significantly affected by a first RZV dose. Grade 3 reactogenicity was associated with a small transient decrease in physical functioning 1-2 days post-dose-1 that resolved by Day 3 post-vaccination.

Funding: GlaxoSmithKline Biologicals SA

Table 1. Mean SF-36 Physical Functioning scores pre- and post-first vaccination by day, reactogenicity grade and symptom type (total vaccinated cohort)

\begin{tabular}{lcccccc}
\hline Day & $\begin{array}{c}\text { Grade } 0 \\
\mathrm{~N}=64\end{array}$ & $\begin{array}{c}\text { Grade 1 or 2 } \\
\mathrm{N}=299\end{array}$ & $\begin{array}{c}\text { Grade 3 } \\
\mathrm{N}=38\end{array}$ & $\begin{array}{c}\text { No symptoms } \\
\mathrm{N}=64\end{array}$ & $\begin{array}{c}\text { Local } \\
\text { symptoms } \\
\mathrm{N}=321\end{array}$ & $\begin{array}{c}\text { Systemic } \\
\text { symptoms } \\
\mathrm{N}=220\end{array}$ \\
\hline Pre-vaccination & & & &
\end{tabular}

\begin{tabular}{llllllll}
\hline Pre-vaccination & & & & & & \\
& & & & & & \\
-7 & 76.8 & 82.8 & 75.5 & 76.8 & 81.8 & 81.8 \\
0 & 82.3 & 84.3 & 75.8 & 82.3 & 83.5 & 82.8 \\
\hline
\end{tabular}

\begin{tabular}{lllllll}
\hline Post-vaccination & & & & & & \\
$\mathbf{1}$ & 84.8 & 84.1 & 65.2 & 84.8 & 82.0 & 79.7 \\
$\mathbf{2}$ & 84.7 & 85.5 & 68.0 & 84.7 & 83.8 & 82.3 \\
$\mathbf{3}$ & 84.9 & 85.7 & 74.8 & 84.9 & 84.5 & 83.7 \\
$\mathbf{4}$ & 84.8 & 85.6 & 75.7 & 84.8 & 84.5 & 83.6 \\
$\mathbf{5}$ & 85.0 & 85.7 & 77.2 & 85.0 & 84.8 & 84.0 \\
$\mathbf{6}$ & 85.0 & 85.7 & 74.7 & 85.0 & 84.5 & 83.7 \\
7 & 83.1 & 85.4 & 75.5 & 83.1 & 84.7 & 82.9 \\
\hline Norms of SF-36 Physical Functioning scores in the US for ages $45-54,55-64,65-74$ and $75-89$ are $0.80,0.78,0.78$ and 0.76,
\end{tabular}
respectively (Fryback et al. Med Care. 2007;45(12):1162-70); High scores represents high level of functioning/quality-of-life; $\mathrm{N}$, total number of vaccinated participants; Reactogenicity grading: 0 (none/normal); 1 (mild); 2 (moderate); 3 (severe; preven

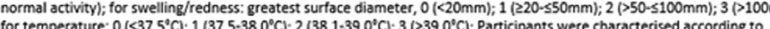
maximum reacogenicity grade repored within 7 days post-dose 1

Disclosures. K. E. Schmader, GSK group of companies: Investigator, Research grant. M. J. Levin, GSK group of companies: Grant Investigator and Scientific Advisor, Consulting fee and Research grant. K. Grupping, GSK group of companies: Employee, Salary. S. Matthews, GSK group of companies: Consultant, Consulting fee. M. El Idrissi, GSK group of companies: Employee and Shareholder, Salary. L. A. Fissette, GSK group of companies: Employee, Salary. C. Fogarty, Medical Research: Investigator, Research grant. N. P. Klein, GSK group of companies: Investigator, Research support. M. Nevarez, GSK group of companies: Investigator, Investigator stipend. K. Uusinarkaus, GSK group of companies: Investigator, Research grant. L. Oostvogels, GSK group of companies: Employee, Salary. D. Curran, GSK group of companies: Employee and Shareholder, GSK shares and Salary.

2490. A Phase 1, Randomized, Observer Blind, Antigen and Adjuvant Dosage Finding Study to Evaluate the Safety and Immunogenicity of an Adjuvanted, Trivalent Subunit Influenza Vaccine in Elderly Subjects $\geq 65$ Years of Age Gillis Otten, $\mathrm{PhD}^{1}$; Vince Matassa, $\mathrm{PhD}^{2}$; Max Ciarlet, $\mathrm{PhD}^{3}$ and Brett Leav, $\mathrm{MD}^{1}$; ${ }^{1}$ Seqirus, Inc., Cambridge, Massachusetts, ${ }^{2}$ Seqirus Pty Ltd., Parkville, Victoria, Australia, ${ }^{3}$ Novartis Vaccines, Cambridge, Massachusetts

Session: 254. Vaccines for the Elderly and Immune Compromised Saturday, October 6, 2018: 12:30 PM

Background. Influenza virus infection in the elderly remains one of the ten leading causes of death. One successful strategy to enhance the magnitude of their influenza vaccine immune response has been the addition of the adjuvant MF59.

Methods. 196 subjects $\geq 65$ years of age were enrolled in a dose ranging study with seven treatment arms to assess the safety and immunogenicity of the current formulation of aTIV compared with aTIV-modified formulations in which the dosage of MF59 was doubled or tripled and/or the dosage of the three influenza virus strains $(\mathrm{A} / \mathrm{H} 1 \mathrm{~N} 1, \mathrm{~A} / \mathrm{H} 3 \mathrm{~N} 2$, and $\mathrm{B})$ was doubled. Vaccine was administered by single or bilateral deltoid inoculations. The antibody responses to all three influenza virus vaccine strains were compared 21 days after a dose or doses of aTIV or aTIV-modified formulations, as measured by hemagglutination inhibition (HI) assay and microneutralization $(\mathrm{MN})$ assay.

Results. In general, $\mathrm{HI}$ and MN titers at Day 22 increased to a greater degree with the dosage of MF59 compared with that of HA (HI presented in Figure 1). This was evident when comparing the $\mathrm{HI}$ and $\mathrm{MN}$ titers where antigen content was a constant $45 \mu \mathrm{g}$, but MF59 dose ranged from $9.75,19.5$ to $29.25 \mathrm{mg}$ in a single vaccine dose (Group 1, 2 and 6, respectively). Generally, the highest titers against all strains were evident with the highest MF59 dose $(29.25 \mathrm{mg})$. The relationship of antigen content and immunogenicity of the vaccine was less apparent when comparing titers between groups in which HA antigen content doubled from 45 to $90 \mu \mathrm{g}$. Administering the dose of MF59 $(19.5 \mathrm{mg})$ and TIV $(90 \mu \mathrm{g})$ into either a single arm or dividing between two arms resulted in comparable titers. The incidence of solicited AEs tended to increase with the dose of MF59 and to a lesser degree, antigen. The majority of solicited AEs were mild to moderate in severity. The number of unsolicited AEs were similar across the different dosages used in this trial.

Conclusion. In elderly subjects $\geq 65$ years of age, increase in MF59 dose is associated with increased immunogenicity against all 3 components of seasonal influenza vaccine.

Figure 1. Geometric mean titer (HI) against influenza A/H3N2/Texas/2012 according to dose of MF59 and $\mathrm{HA}$.

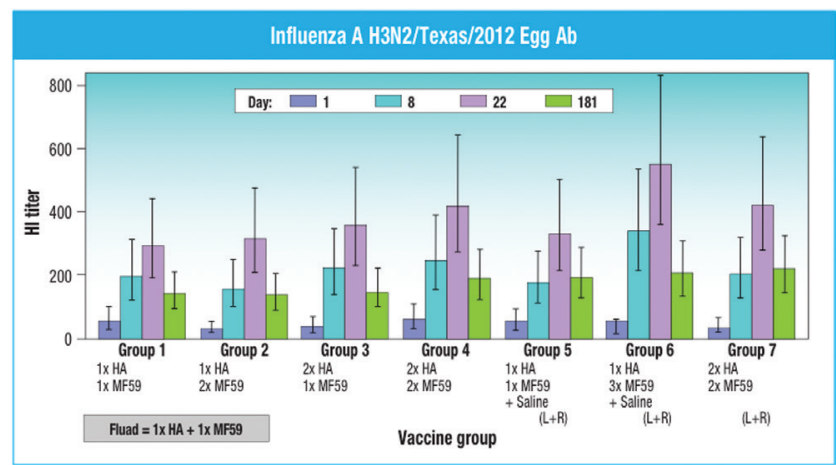

Disclosures. G. Otten, Seqirus: Employee, Salary. V. Matassa, Seqirus: Employee Salary. M. Ciarlet, Novartis Vaccines: Employee, Salary. B. Leav, Seqirus: Employee and Shareholder, Salary.

2491. Post-Exposure Prophylaxis With Ribavirin Plus Lopinavir/Ritonavir for Middle East Respiratory Syndrome in Healthcare Workers

So Yeon Park, $\mathrm{MD}, \mathrm{PhD}^{1}$; Jin Seo Lee, $\mathrm{MD}^{2}$; Jungok Kim, $\mathrm{MD}, \mathrm{PhD}^{3}$; Eun-Jeong Joo, $\mathrm{MD}, \mathrm{PhD}^{4}$; Joong Sik Eom, $\mathrm{MD}^{5}$ and Kyong Ran Peck, $\mathrm{MD}^{6}$; ${ }^{1}$ Infectious Diseases, Kangdong Sacred Heart Hospital Hallym University School of Medicine, Seoul, Korea, Republic of (South), ${ }^{2}$ Kangdong Sacred Heart Hospital, Hallym medica school, SEOUL, Korea, Republic of (South), ${ }^{3}$ Chungnam National University Hospital, Daejeon, Korea, Republic of (South), ${ }^{4}$ Division of Infectious Diseases, Kangbuk Samsung Hospital, Sungkyunkwan University School of Medicine, Seoul Korea, Republic of (South), ${ }^{5}$ Infectious Diseases, Gachon University Gil Medical Center, Seoul, Korea, Republic of (South), ${ }^{6}$ Division of Infectious Diseases, Samsung Medical Center, Sungkyunkwan University School of Medicine, Seoul, Korea, Republic of (South)

Session: 255. Virology Potpourri

Saturday, October 6, 2018: 12:30 PM

Background. In 2015, an outbreak of Middle East Respiratory Syndrome coronavirus (MERS-CoV) infection occurred in South Korea involving 186 patients, 39 of whom were healthcare workers (HCWs) exposed to the infection. An effective post-exposure prophylaxis (PEP) strategy may limit the spread of infection; however, there is no consensus regarding PEP for MERS-CoV infection. In this study, we assessed (1) the efficacy of oral ribavirin and lopinavir/ritonavir as PEP for HCWs exposed to patients with severe MERS-CoV pre-isolation pneumonia, and (2) safety of the PEP regimen.

Methods. We retrospectively enrolled $43 \mathrm{HCW}$ s with high-risk exposure to MERS-CoV from 5 hospitals affected during this outbreak in South Korea. The rate of MERS-CoV infection was compared between 22 workers at 1 hospital who received $\mathrm{PEP}$ consisting of oral ribavirin and lopinavir/ritonavir after exposure to patients with severe MERS-CoV pre-isolation pneumonia and 21 workers at other hospitals who did not receive PEP.

Results. Six workers (14\%) developed MERS-CoV infection; all of these subjects belonged to the non-PEP group. The attack rate was lower in the PEP group compared with the non-PEP group $(0 \%$ vs. $28.6 \%$; Odds ratio $=0.405,95 \%$ confidence interval $=0.274-0.599 ; P=0.009)$. The most commonly reported side effects of PEP therapy were nausea and diarrhea, but there were no severe adverse effects associated with PEP therapy. 
Conclusion. PEP with a combination of oral ribavirin and lopinavir/ritonavir appears to be effective and generally safe for preventing MERS-CoV infection after high-risk exposure in healthcare workers.

Disclosures. All authors: No reported disclosures.

2492. Clinical, Virologic, and Immunologic Characteristics of Zika Virus Infection in a Cohort of US Patients

Hana M. El Sahly, MD, FIDSA ${ }^{1}$; Rodion Gorchakov, $\mathrm{MS}^{2}$; Kristy Murray, DVM, $\mathrm{PhD}^{2}$; Shital Patel, MD ${ }^{3}$; Robert L. Atmar, MD ${ }^{4}$; Wendy Keitel, MD, FIDSA ${ }^{5}$; Daniel Hoft, MD, $\mathrm{PhD}^{6}$; Jill Barrett, $\mathrm{MPH}^{7}$; Jason Bailey, $\mathrm{PhD}^{8}$; Nadine Rouphael, $\mathrm{MD}^{9}$; Srilatha Edupuganti, $\mathrm{MD}^{10}$; Vanessa Raabe, $\mathrm{MD}^{9}$; Henry Wu, MD ; Jessica Fairley, $\mathrm{MD}^{9}$; Muktha Natrajan, $\mathrm{PhD}^{11}$; Lilin Lai, $\mathrm{MD}^{12}$ and Mark J. Mulligan, MD, FIDSA ${ }^{12} ;{ }^{1}$ Molecular Virology and Microbiology, Baylor College of Medicine, Houston, Texas, ${ }^{2}$ Pediatrics-Tropical Medicine, Texas Children Hospital, Baylor College of Medicine, Houston, Texas, ${ }^{3}$ Baylor College of Medicine, Department of Medicine, Houston, Texas, ${ }^{4}$ Medicine, Baylor College of Medicine, Houston, Texas, ${ }^{5}$ Molecular Virology \& Microbiology and Medicine, Baylor College of Medicine, Houston, Texas, ${ }^{6}$ Saint Louis University Health Sciences Center, St. Louis, Missouri, ${ }^{7}$ The EMMES Corp, Rockville, Maryland, ${ }^{8}$ Emmes Corporation, Rockville, Maryland, ${ }^{9}$ Emory University, Atlanta, Georgia, ${ }^{10}$ Medicine/Infectious Diseases, Emory University School of Medicine, Decatur, Georgia, ${ }^{11}$ Emory University Hope Clinic, Atlanta, Georgia, ${ }^{12}$ Hope Clinic-Emory University, Decatur, Georgia

Session: 255. Virology Potpourri

Saturday, October 6, 2018: 12:30 PM

Background. The clinical, virologic and immunologic characteristics of Zika virus (ZIKV) infections in US patients are poorly defined.

Methods. US patients with suspected Zika virus (ZIKV) infection were enrolled and clinical data and specimens were prospectively collected. Body fluids were tested for ZIKV RNA by PCR and blood was tested using serologic and cellular immune assays. Findings from those with confirmed ZIKV infections (cases) and ZIKVnegative controls were compared.

Results. We enrolled 45 cases and 14 controls. The most commonly reported symptoms among cases and controls were maculopapular rash $(97.8 \%$ and $81.8 \%)$, fatigue $(86.7 \%$ and $81.8 \%)$ and arthralgia $(82.2 \%$ and $54.5 \%)$, respectively. The sensitivity and duration of detection by PCR were highest in whole blood samples ( $94 \%$ of 35 cases who had samples collected up to day 79 post illness onset were positive); strikingly, $84 \%$ of those were still positive at $65-79$ days post illness onset (Figure 1). ZIKV neutralizing antibodies were detected in all cases and none of the controls, and titers were significantly higher in dengue virus (DENV)-experienced subjects than in DENV-naïve ones (Figure 2). Among cases, anti-ZIKV IgG antibodies were also significantly higher in DENV-experienced patients, while anti-ZIKV IgM antibodies were no higher in DENV-experienced compared with naïve ones. Using intracellular cytokine staining, the highest frequencies of T cells producing IFN- $\gamma$, IL- 2 and/or TNF- $a$ were against the NS1, NS3, and NS5 proteins for CD4+ T cells, and against the E, NS3, and NS5 proteins for CD8+ T cells (Figure 3).

Conclusion. Detection of ZIKV RNA was more frequent and much more prolonged in whole blood samples compared with other body fluids. Diagnostic molecular assays on this easily obtained fluid should be prioritized for point-of-care development. Robust cellular responses to E, NS3 and NS5 proteins could have implications for vaccine development.

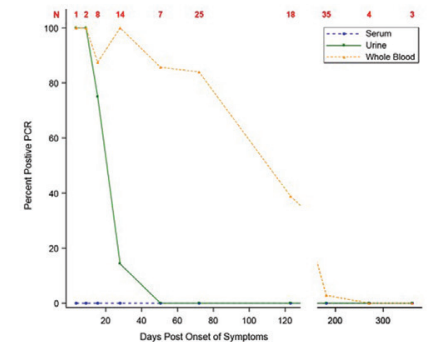

Figure 1. Percent of Zika virus infected patients with a positive PCR in whole blood, urine and serum by day post onset of symptoms. N represents the number of subjects with the specified body fluid at the specified time point.

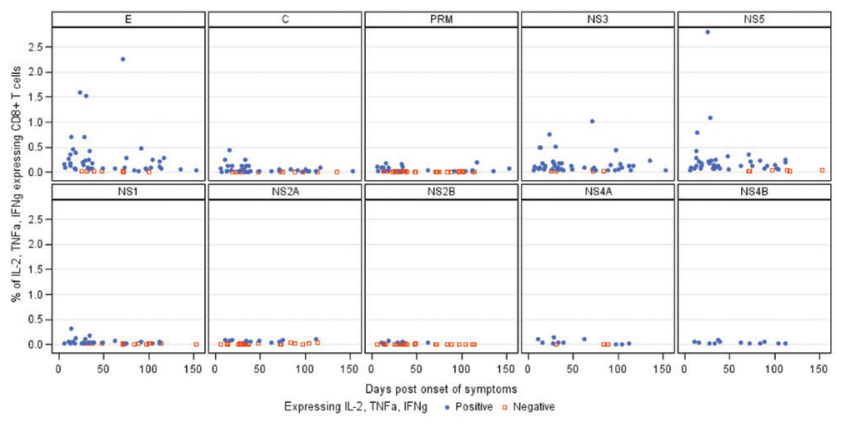

Figure 2. Percent of CD8+T cells expressing IL-2, TNF-a and IFN-g following stimulation with the specified proteins in cases (positive) and controls (negative).

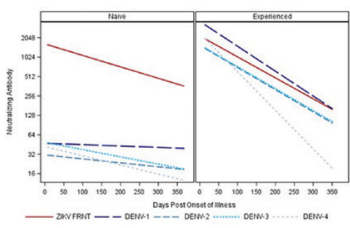

Figure 3. Neutralizing antibody titers by days post onset of illness in Dengue virus-naïve and experienced patients with confirmed Zika virus infection. FRNT=focus reduction neutralizing test.

Disclosures. R. L. Atmar, Takeda Vaccines, Inc.: Investigator, Research grant.

\section{Marburg Virus Disease: Virulence of Angola vs. Musoke Strain in}

Cynomolgus Macaques

Paul Blair, $\mathrm{MD}, \mathrm{MSPH}^{1}$; Maryam Keshtkar-Jahromi, $\mathrm{MD}, \mathrm{MPH}^{1}$; Kevin Psoter, $\mathrm{PhD}$,

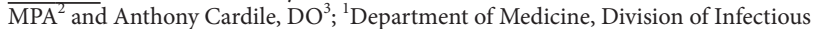

Diseases, Johns Hopkins University School of Medicine, Baltimore, Maryland, ${ }^{2}$ Johns

Hopkins Bayview Medical Center, Baltimore, Maryland, ${ }^{3}$ The United States Army

Medical Research Institute for Infectious Diseases, Frederick, Maryland

Session: 255. Virology Potpourri

Saturday, October 6, 2018: 12:30 PM

Background. From 2004 to 2005, an outbreak of Marburg virus, a filovirus, in Angola led to a case-fatality rate of 90 percent. However, little information is available regarding the virulence of the Angola strain from this outbreak compared with the virulence of other strains. Therefore, we sought to assess time to selected outcomes in non-human primates (NHPs) experimentally infected with either Angola or Musoke Marburg strains.

Methods. Between 2012 and 2017, nine therapeutic trials at the US Army Medical Research Institute of Infectious Diseases were conducted in Macaca fascicularis monkeys challenged with 1 to 10,000 plaque forming units of Marburg virus administered intramuscularly. The current study population was comprised of 90 control NHPs, of which, 61 were administered Angola strain in four separate trials and 29 with Musoke strain in five trials. Clinical responses including development of rash and oral intake were collected following infection. The primary outcome of interest was time to death or euthanasia post-inoculation between strains evaluated using Cox proportional haz ards regression. Secondary endpoints included time to development of a petechial rash and time to decreased appetite.

Results. Following Marburg virus challenge, all NHPs died and most NHPs experienced decreased food consumption (97\%), and petechial rash (96\%). The median time to death for Angola-infected NHPs was 8.9 days (25th, 75 th percentiles: 7.9, 9.3), whereas Musoke-infected NHPs survived for a median of 10.0 days (25th, 75th percentiles: 9.0, 10.9) (Figure 1). Irrespective of strain, petechial rash was preceded by decreased food consumption by 0.7 days (SD 1.5) on average. Angola strain was associated with statistically significant earlier death (adjusted $\mathrm{HR}=21.8 ; 95 \%$ CI: 8.9, 53.2), earlier development of petechiae (adjusted $\mathrm{HR}=17.6$; 95\% CI: 7.0, 44.5) and earlier loss of appetite (adjusted HR $=5.8 ; 95 \%$ CI: $2.9,11.7)$

Conclusion. This was the first study to compare survival and clinical characteristics in NHPs between these strains. Despite sharing the similar genetic lineage, our data strongly supports increased virulence of Angola strain compared with Musoke strain. Pathophysiological mechanisms involved in increased virulence require further study.

Figure 1. Kaplan Meier survival curves in Cynomolgus macaques for A) overall time to death petechial rash, and decreased food consumption $(n=77) ; B)$ time to death by strain $(n=90) ; C$ ) time to decreased food consumption by strain $(n=79) ; D)$ time to petechial rash by strain $(n=77)$
A.

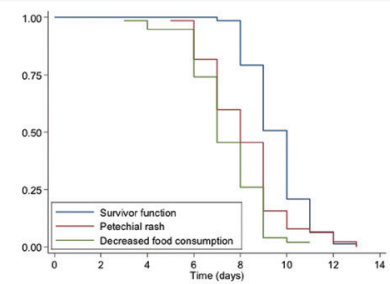

C.

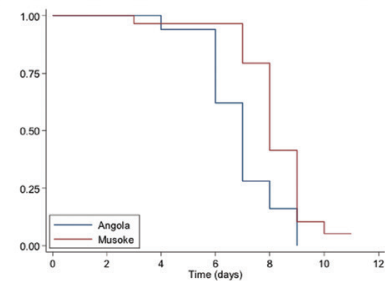

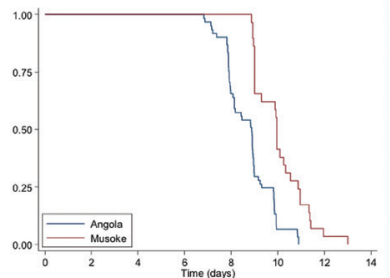

D.

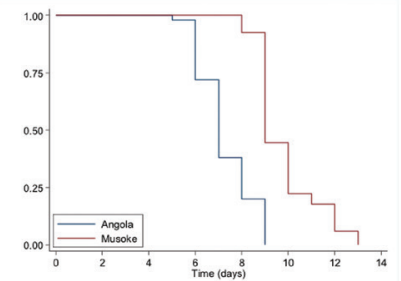

Disclosures. All authors: No reported disclosures. 